\title{
Increased Risk of Rheumatoid Arthritis-Related Pulmonary Disease as a Results of Serum Anticitrullinated Protein Antibody Positivity \\ Samia Abdel-Hamid Abdel-Mageed ${ }^{1}$, Eldesoky E. Foda ${ }^{2}$, \\ Emam Mohamed Abdel-Azeez ${ }^{3}$, Seham Abdallah Elazab ${ }^{1 *}$
}

${ }^{1}$ Department of rheumatology and Rehabilitation, ${ }^{2}$ Department of Internal Medicine and Immunology and Allergy Center, ${ }^{3}$ Department of Radiology, Faculty of Medicine, Al-Azhar University, Cairo, Egypt

*Correspondence author: Seham Abdallah Elazab, Mobile: (+20)01000263048, E-mail: s.abdallah.azab@gmail.com

\begin{abstract}
Background: there is increasing evidence to indicate that autoimmunity as well as inflammatory reactions occur systemically before development of any clinical sign of joint disease in patients with anti-citrullinated protein antibody (ACPA)-positive rheumatoid arthritis (RA).

Objective: The aim of the work was to investigate the potential predictive value of anti-ccp3 for interstitial lung disease association with RA and to discriminate RA patient who are at potential risk to develop methotrexate (MTX) induced pulmonary toxicity.

Patients and methods: This cross-sectional study included a total of 50 RA patients who were diagnosed according to the 2010 American College of Rheumatology / the European League Against Rheumatism classification criteria for Rheumatoid Arthritis (ACR/EULAR 2010 criteria) for detection of pulmonary manifestations attending at Physical medicine, Rheumatology and Rehabilitation Outpatient Clinics of Al-Azhar University Hospitals.

Results: there was statistically significant difference between patients with and without interstitial lung disease (ILD), in high-resolution computed tomography (HRCT) as regards to Tiffeneau-Pinelli index (FEV1/FVC ratio), except for FEV1/FVC between patients with and without ILD in HRCT, forced expiratory volume in first second (FEV1), and forced vital capacity (FVC) are often decreased because of the reduction in lung volume, but the FEV1to-FVC ratio is maintained or increased in ILD.

Conclusion: RA patients without chest symptoms should not be neglected, pulmonary function testing and HRCT should be done routinely to pick up early ILD cases.
\end{abstract}

Keywords: Rheumatoid arthritis, pulmonary disease, PCPA.

\section{INTRODUCTION}

Rheumatoid arthritis (RA) is a chronic, systemic, inflammatory disease that involves synovial joints and other organs and extra-articular involvements associated with impairment in physical function, higher morbidity, and premature mortality ${ }^{(1)}$.

Rheumatoid arthritis affects $\sim 1 \%$ of the population in developed countries. The incidence and prevalence of rheumatoid arthritis in developing countries is thought to be lower, but is difficult to quantify. Although joint disease is the main presentation, there are a number of extra-articular manifestations including subcutaneous nodule formation, vasculitis, inflammatory eye disease and lung disease ${ }^{(2)}$.

Of these manifestations, lung disease is a major contributor to morbidity and mortality. interstitial lung disease is the only complication of RA increasing in prevalence and accounts for approximately $6 \%$ of all RA deaths ${ }^{(3)}$.

It is critical for the pulmonologist to assess for systemic and articular signs and symptoms of connective tissue disease when evaluating a patient with pulmonary disease of unknown etiology as patients may initially present with pulmonary symptoms ${ }^{(2)}$.

There are a variety of pulmonary manifestations of rheumatoid arthritis, including pulmonary parenchymal disease (interstitial lung disease (ILD)) and inflammation of the pleura (pleural thickening and effusions), airways and pulmonary vasculature (vasculitis and pulmonary hypertension). These changes may reflect chronic immune activation, increased susceptibility to infection (often related to immunomodulatory medications) or direct toxicity from disease modifying or biological therapy. Prognosis varies depending on the type and severity of involvement ${ }^{(2)}$.

While the rate of some extra-articular manifestations of rheumatoid arthritis have decreased with improvements in therapy, the incidence of ILD has remained fairly stable if not increased, Whether this reflects an increase in detection or is the result of druginduced lung disease with more aggressive use of antirheumatic agents is not entirely clear ${ }^{(4)}$.

Methotrexate (MTX) is the most frequently used therapy in rheumatoid arthritis (RA) and is used widely in many other autoimmune disorders. Pulmonary toxicity has been reported in patients receiving lowdose MTX, and acute hypersensitivity pneumonitis is the most feared complication.

It has also been suggested that chronic pulmonary fibrosis can be caused by MTX, but specific risk factors for the development of pulmonary toxicity in patients treated with low- dose MTX have never been identified. Some hypotheses concerning RA pathogenesis suggest that One of the consequences of the formation of anti-cyclic citrullinated peptide antibodies(anti-CCP), which are observed in around 55- 
$69 \%$ of patients with RA Is the development of extra- articular manifestations ${ }^{(5)}$.

The aim of the current work was to investigate the potential predictive value of anti-ccp3 for interstitial lung disease association with RA and to discriminate RA patient who are at potential risk to develop MTX induced pulmonary toxicity.

\section{PATIENTS AND METHODS}

This cross-sectional study included a total of 50 RA patients who were diagnosed according to the 2010 American College of Rheumatology / the European $>$ League Against Rheumatism classification criteria for Rheumatoid Arthritis (ACR/EULAR 2010 criteria) ${ }^{(6)}$ for detection of pulmonary manifestations attending at Physical medicine, Rheumatology and Rehabilitation Outpatient Clinics of Al-Azhar University Hospitals. Approval of the ethical committee and a written informed consent from all the subjects were obtained.

This study was conducted between May 2016 to March 2017.

They were 2 males (4\%) and 48 females (96\%) their ages ranged between 21 to 70 years with mean \pm SD age of $45.76 \pm 12.28$ years.

\section{Exclusion criteria:}

1. Any other autoimmune diseases.

2. Patients with malignancies.

\section{They were subjected to:}

1- Full history taking: ACR patient history form

2. Full clinical examination and disease activity assessment for RA patients by disease activity score 28 $\left(\right.$ DAS28) ${ }^{(7)}$.

\section{3-Examination:}

A) General examination:

- Vital signs:

- Head and neck examination:

- Upper limb examination:
Lower limb examination:

- Cardiovascular examination:

In order to diagnose pulmonary hypertension, cor-pulmonale, pericarditis, systemic hypertension and also to exclude other causes of dyspnea of cardiac origin e.g. rheumatic heart disease, congenital heart disease.

$>$ Chest examination:

a. Inspection: *Shape of chest.

$>$ Respiratory movements.

$>$ Pulsations of the different areas e.g. epigastric, left parasternal, and pulmonary area.

b. Palpation: *Confirm respiratory movements, and pulsations.

- Chest expansion.

- Palpation of the trachea.

- Palpable rhonchi.

- Tactile vocal fremitus.

- Localized tenderness.

c. Percussion: *anterior chest wall. d.

$>$ Posterior chest wall.

$>\quad$ Lateral chest wall.

e. Auscultation: *Breathe sounds.

$>$ Adventitious sounds.

$>$ Abdominal examination: For organomegaly or ascites.

$>$ Neurological examination.

Clinical assessment of disease activity of $R A$ patients:

The Disease Activity Score 28 (DAS28) includes the 28-swollen joint count (28-SJC) and the 28tender joint count (28-TJC) Fig. (1), in addition to patient global assessments of disease activity on a VAS, and ESR or CRP. The scores according to EULAR criteria are: $\leq 2.6$ for remission, $>2.6$ and $\leq 3.2$ for low disease activity, $>3.2$ and $\leq 5.1$ for moderate disease activity and $>5.1$ for high disease activity ${ }^{(8)}$.

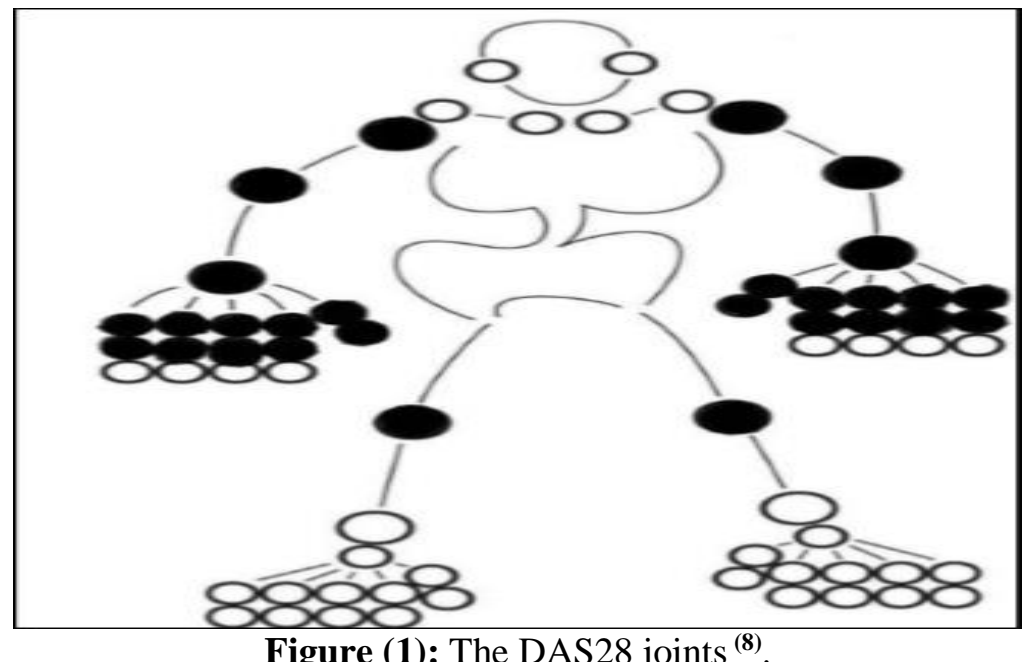

Figure (1): The DAS28 joints ${ }^{(8)}$. 
3. Plain X-ray hand and feet as a routine.

4. Laboratory investigation: Anti-cyclic citrullinated peptide antibodies third-generation (anti-CCP3) serum titer. Erythrocyte sedimentation rate (ESR), $\mathrm{C}$ reactive protein (CRP).

5. Chest High- resolution computed tomography (HRCT): The CT examinations were obtained on a 160 multi slice Aquilion prime Toshipa in Alzahraa university hospital radiology department.

The technique used for assessing pulmonary functions was as follows:

The subject was asked to put the mouthpiece, starting at the end of a normal exhalation. The subject was instructed to breath in a normal tidal range, then to inhale to total lung capacity and exhale as hard and fast as possible to residual volume RV. This maneuver was repeated three times for each patient to obtain the best results. A restrictive pattern is indicated by an FVC less than $80 \%$, an obstructive defect is indicated by a low forced expiratory volume in one second/forced vital capacity (FEV1/FVC) ratio, which is defined as less than $70 \%$ if both the FEV1/FVC ratio and the FVC are low, the patient has a mixed defect ${ }^{(9)}$.

\section{Statistical analysis}

Data were analyzed using Statistical Program for Social Science (SPSS) version 20.0. Quantitative data were expressed as mean \pm standard deviation (SD). Qualitative data were expressed as frequency and percentage.

\section{The following tests were done:}

- Independent-samples t-test of significance was used when comparing between two means.

- Chi-square $\left(\mathrm{X}^{2}\right)$ test of significance was used in order to compare proportions between two qualitative parameters.

- Spearman's rank correlation coefficient (rs) was used to assess the degree of association between two sets of variables if one or both of them was skewed.

- Probability (P-value)

- P-value $<0.05$ was considered significant.

- P-value $<0.001$ was considered as highly significant.

- P-value $>0.05$ was considered insignificant.

\section{RESULTS}

In a cross-sectional study, 50 RA patients was randomly chosen fulfilling the ACR/EULAR 2010 classification criteria of RA Aletaha et al. (6) for detection of pulmonary manifestations. All patients were assessed by clinical examination, laboratory investigations in the form of ESR, CRP, RF and anti CCP3 serum titer. Chest HRCT was done for detection of pathological pulmonary changes. Office spirometer was also done for evaluation of pulmonary function in the study group.
Study included 48 female (96\%) and 2 male (4\%), age ranged from 21 to 70 with mean $45.76 \pm 12.28$, Disease duration ranged from 1 to 30with mean (9.76 \pm 6.63$)$ Table (1).

Table (2): Demographic data of RA patients.

\begin{tabular}{|l|c|}
\hline & Total $(\mathbf{N}=50)$ \\
\hline Age (years) & $21-70(45.76 \pm 12.28)$ \\
\hline Sex & \\
\hline Female & $48(96 \%)$ \\
\hline Male & $2(4 \%)$ \\
\hline Disease duration & $1-30(9.76 \pm 6.63)$ \\
\hline
\end{tabular}

With respect to the clinical data of RA patients (Table 2), TJC ranged from 0 to 26 mean [8.26 \pm 6.31 ], SJC ranged from 0 to 14 mean [2.58 \pm 3.67$]$, VAS ranged from 10 to 70 mean [35.88 \pm 17.70$]$, DAS 28 ranged from 2.4 to 7.8 mean $[4.7 \pm 1.28], 2$ patients were in remition with DAS 2.4and 2.6, 2 patients showed low disease activity DAS 2.9 and3.2, 31 patients showed moderate disease activity with DAS range $>3.2$ and $\leq 5.1,15$ patients with high disease activity with DAS $>5.1,22(44 \%)$ suffered from chest symptoms. 15 patients $30 \%$ suffered from sicca complex, 8 patients were diabetic (16\%),13 patients (26\%)were hypertensive.

Table (3): Clinical features of RA patients.

\begin{tabular}{|l|c|}
\hline Clinical Data & Total $(\mathbf{N}=\mathbf{5 0})$ \\
\hline Chest symptoms & $22(44 \%)$ \\
\hline DAS 28 & $2.4-7.81[4.73 \pm 1.28]$ \\
\hline TJC & $0-26[8.26 \pm 6.31]$ \\
\hline SJC & $0-14[2.58 \pm 3.67]$ \\
\hline VAS & $10-70[35.88 \pm 17.70]$ \\
\hline DM & $8(16 \%)$ \\
\hline HTN & $13(26 \%)$ \\
\hline Sicca Complex & $15(30 \%)$ \\
\hline
\end{tabular}

HRCT chest of all patients showed 27(54\%) patients with normal HRCT, 23(46\%) with abnormal HRCT, 19 (38\%) patients with evidence of ILD, 8 patients $(16 \%)$ with pulmonary nodules, 5 patients showed pulmonary nodules with ILD.3pateints showed pulmonary nodules with normal interstitium, nodules size ranged from $5 \mathrm{~mm}$ to $2 \mathrm{~cm}$ in diameter, 3 patients (6\%) showed Emphysematous changes Table (3).

Table (3): HRCT chest findings of RA patients.

\begin{tabular}{|l|c|}
\hline CT chest finding & Total $(\mathbf{N}=50)$ \\
\hline Normal HRCT & 27 \\
\hline Abnormal HRCT & 23 \\
\hline ILD ONLY & 13 \\
\hline Pulmonary Nodule ONLY & 3 \\
\hline Pulmonary Nodule with ILD & 5 \\
\hline
\end{tabular}



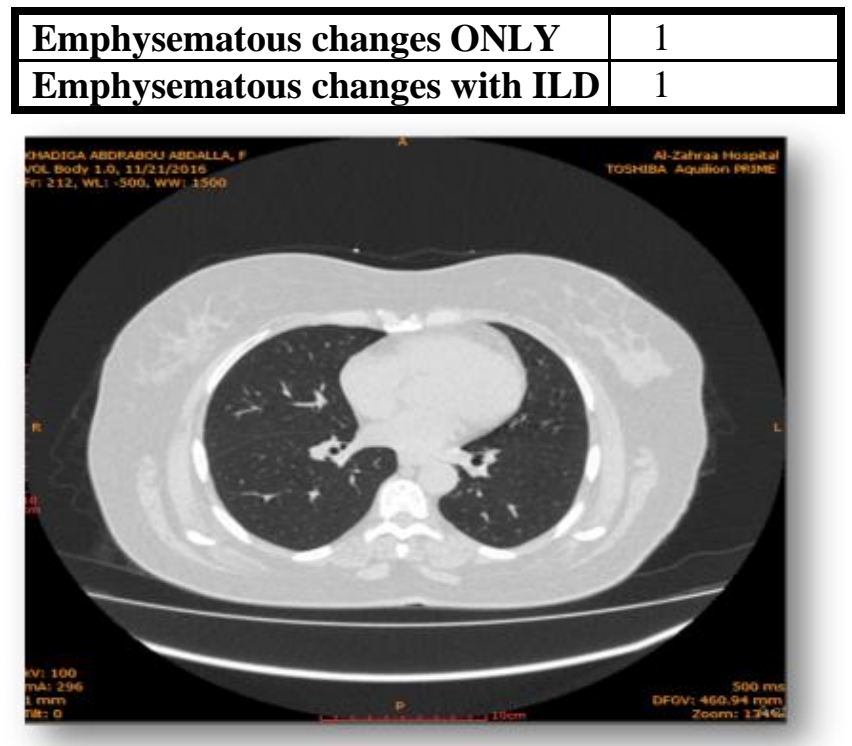

Figure (2): Normal chest HRCT study of RA female patient

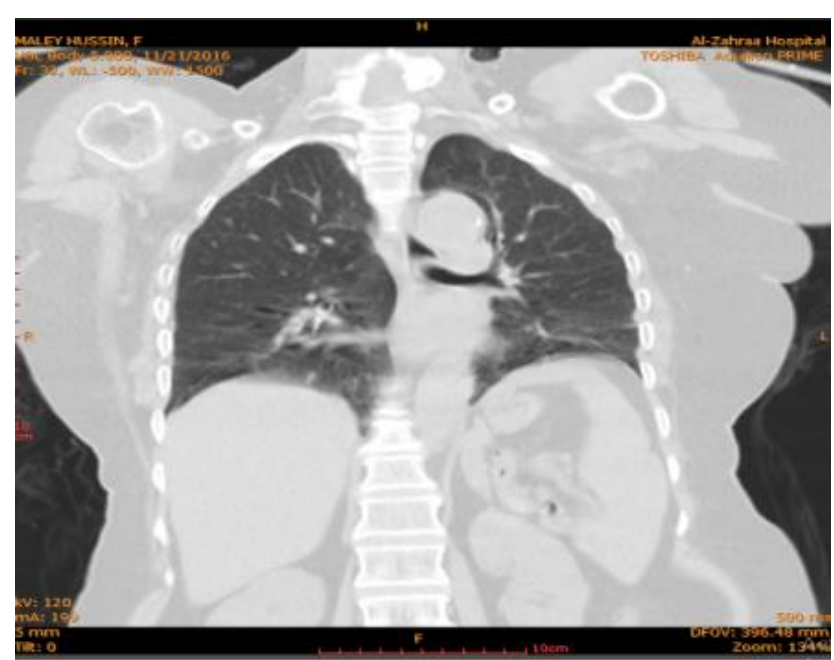

Figure (3): Chest HRCT study of RA female patient showing bilateral basal ground glass veiling of both lower lung fields

Patients with normal HRCT despite presence of chest complaint in the form of mild dyspnea on exertion showed increased incidence of other comorbidities including hypertension, diabetes mellitus and reduced blood hemoglobin level Table (4).
Table (4): comorbidities associated with RA patients including HTN, DM and anemia.

\begin{tabular}{|c|c|c|c|c|}
\hline & $\begin{array}{c}\text { Abnormal } \\
\text { HRCT }\end{array}$ & $\begin{array}{c}\text { Normal CT } \\
\text { with } \\
\text { chest } \mathrm{S}\end{array}$ & $\begin{array}{l}\text { Normal } \\
\text { CT }\end{array}$ \\
\hline & & No. $=23$ & No. $=7$ & No. $=20$ \\
\hline \multirow{2}{*}{ DM } & Negative & $20(86.9 \%)$ & $3(42.8 \%)$ & $9(95.0 \%)$ \\
\hline & Positive & $3(13 \%)$ & $4(57 \%)$ & $1(5.0 \%)$ \\
\hline \multirow{2}{*}{ HTN } & Negative & $18(78.3 \%)$ & $2(28.6 \%)$ & $17(85 \%)$ \\
\hline & Positive & $5(21.7 \%)$ & $5(71.4 \%)$ & $3(15 \%)$ \\
\hline \multirow[b]{2}{*}{ HB } & Mean \pm & $9.43 \pm 0.86$ & $8.50 \pm 0.96$ & $\begin{array}{c}9.93 \pm \\
135\end{array}$ \\
\hline & $\begin{array}{l}\mathrm{SD} \\
\text { Range }\end{array}$ & $8-11$ & $7-9.5$ & $\begin{array}{c}1.35 \\
7-12.5\end{array}$ \\
\hline
\end{tabular}

As regards to clinical data for patients with abnormal HRCT. Among those patients 15patients (65\%) has chest symptoms, 8 patients (34\%)did not show any chest symptoms, DAS 28 ranged from 3.2 to 7.5 ,VAS range from 10 to 70 , swolen joint count ranged from 0 to 14,tender joint count ranged from 0 to 22,9 patients(39\%) were positive for Sicca complex, 14patients $(60 \%)$ were negative for Sicca complex Table (5).

Table (5): Clinical data for RA patients with abnormal HRCT.

\begin{tabular}{|l|l|c|}
\hline \multicolumn{2}{|c|}{ Clinical data } & Abnormal HRCT \\
\cline { 3 - 3 } TJC & Median(IQR) & No. $=\mathbf{2 3}$ \\
& Range & $10(6-14)$ \\
& Median(IQR) & $2-22$ \\
\multirow{2}{*}{ SJC } & Range & $0-14$ \\
\hline \multirow{2}{*}{ VAS } & Mean \pm SD & $40.87 \pm 19.92$ \\
& Range & $10-70$ \\
\hline \multirow{2}{*}{ DAS 28} & Mean \pm SD & $5.32 \pm 1.25$ \\
& Range & $3.2-7.5$ \\
\hline Chest & Negative & $8(34.8 \%)$ \\
symptoms & Positive & $15(65.2 \%)$ \\
\hline Sjogren & Negative & $14(60.9 \%)$ \\
syndrome & Positive & $9(39.1 \%)$ \\
\hline
\end{tabular}

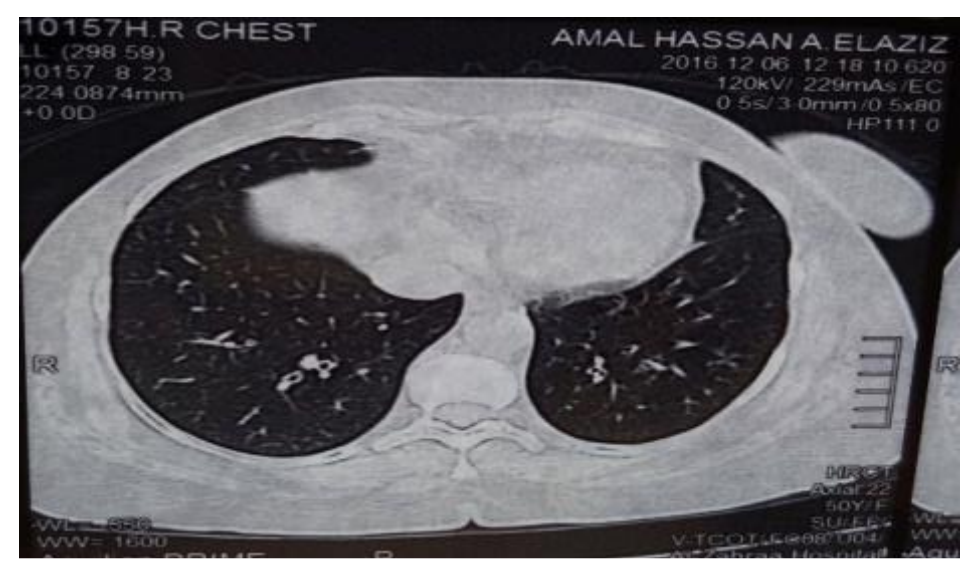


Figure (4): Chest HRCT study of RA female patient showing bilateral emphysematous changes

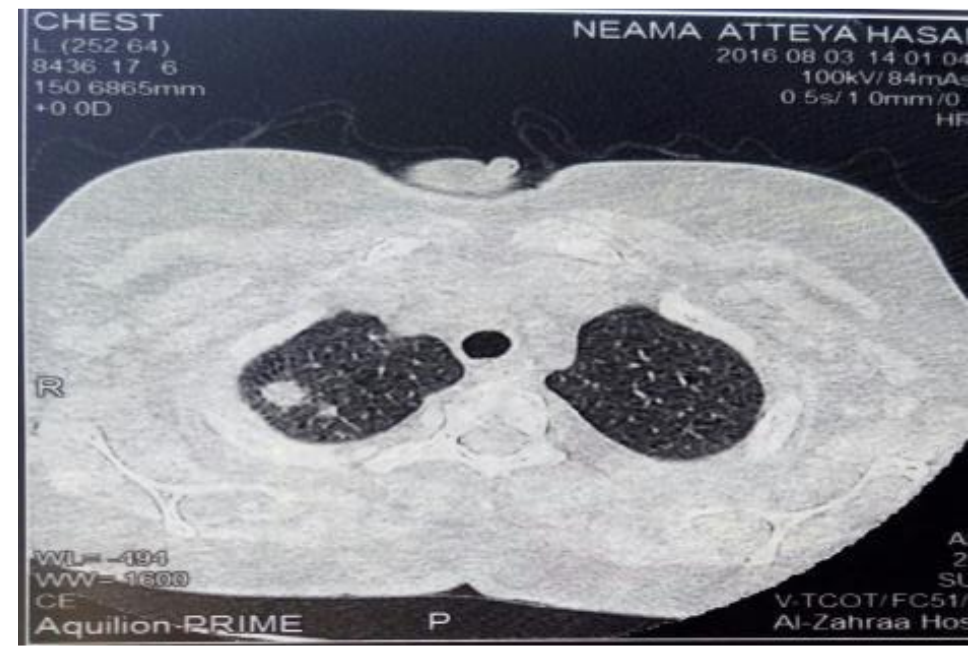

Figure (5): Chest HRCT study of RA female patient showing apical medium sized nodules.

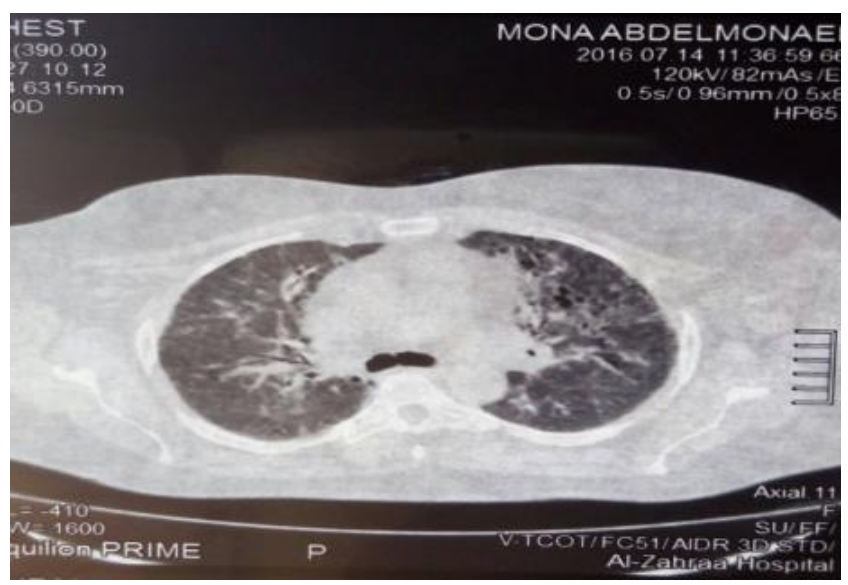

Figure (6): Chest HRCT study of RA female patient showing bilateral traction bronchiectasis with honeycombing.

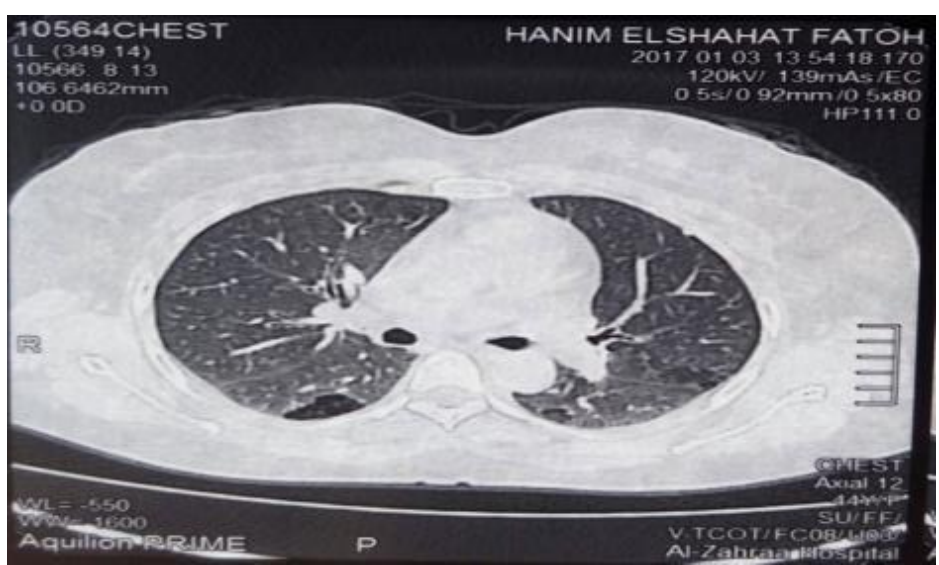

Figure (7): Chest HRCT study of RA female patient showing bilateral basal ground glass veiling of both lower lung fields. 


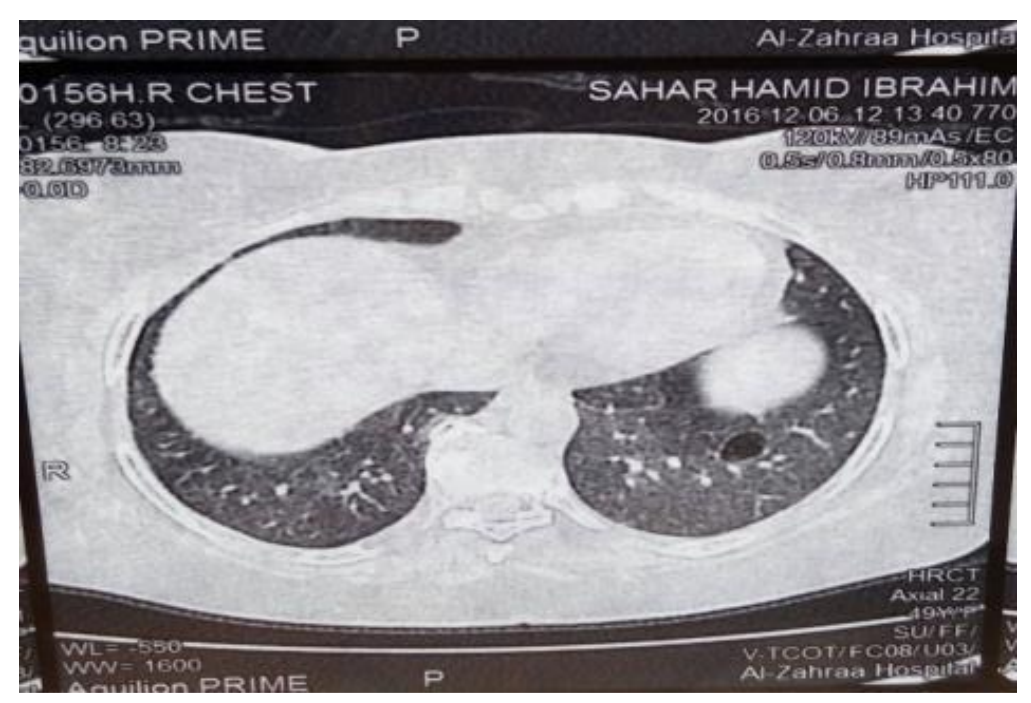

Figure (8): Chest HRCT study of RA female patient showing bilateral basal ground glass veiling of both lower lung fields with unilateral cyst affecting right side.

On Comparing RA patients with and without ILD according to Laboratory data there was statistically significant difference between RA patients with andwithout ILD as regards to Anti-CCP titer(medians 100 versus $20 \mathrm{U} / \mathrm{mL}$ ) (p-value 0.043), RF positivity(p-value 0.031 ). But there was no statistically significant difference as regards to patient ESR level and CRP Table (6).

Table (6): Comparison between RA with and without ILD according to laboratory data.

\begin{tabular}{|c|c|c|c|c|}
\hline \multirow{2}{*}{ Laboratory data } & \multicolumn{2}{|c|}{ ILD [HRCT Score] } & \multirow{2}{*}{ t/x2* } & \multirow{2}{*}{ p-value } \\
\cline { 2 - 4 } & RA without ILD & RA with ILD & & \\
\hline ESR & $33.38 \pm 17.01$ & $43.38 \pm 21.94$ & 1.816 & 0.076 \\
\hline CRP & $20(64.5 \%)$ & $13(68.4 \%)$ & $0.080^{*}$ & 0.777 \\
\hline RF & $19(61.3 \%)$ & $17(89.5 \%)$ & $4.641^{*}$ & 0.031 \\
\hline Anti-CCP titer [Median (IQR)] & {$[20(298)]$} & {$[100(390)]$} & -3.483 & 0.043 \\
\hline
\end{tabular}

On Comparing RA patients with and without ILD according to clinical data there was statistically significant difference between RA patients with and without ILD as regards to presence or absence of chest symptoms ( $p$-value 0.006), DAS 28 (p-value 0.027), But there was no statistically significant difference as regards to other clinical parameters Table (7).

Table (7): Comparison between RA with and without ILD according to clinical data.

\begin{tabular}{|l|c|c|c|c|}
\hline \multirow{2}{*}{ Clinical data } & \multicolumn{2}{|c|}{ ILD [HRCT] } & \multirow{2}{*}{ t/x2* } & \multirow{2}{*}{ p-value } \\
\cline { 2 - 3 } & RA without ILD & RA with ILD & & \\
\hline Tender joint count & $6.97 \pm 6.10$ & $10.05 \pm 6.30$ & 1.739 & 0.088 \\
\hline Swollen joint count & $2.17 \pm 3.40$ & $3.14 \pm 4.03$ & 0.921 & 0.362 \\
\hline VAS & $34.10 \pm 16.17$ & $38.33 \pm 19.77$ & 0.831 & 0.410 \\
\hline DAS 28 & $4.39 \pm 1.21$ & $5.19 \pm 1.26$ & 2.285 & 0.027 \\
\hline Sjogren syndrome & $8(25.8 \%)$ & $7(36.8 \%)$ & $0.683^{*}$ & 0.409 \\
\hline Chest symptoms & $9(29 \%)$ & $13(68.4 \%)$ & $7.417^{*}$ & 0.006 \\
\hline
\end{tabular}

Table (8): Comparison between RA with and without ILD according to pulmonary function test.

\begin{tabular}{|l|c|c|c|c|}
\hline \multirow{2}{*}{ Pulmonary function test } & \multicolumn{2}{|c|}{ ILD [HRCT Score] } & \multirow{2}{*}{ t-test } & \multirow{2}{*}{ p-value } \\
\cline { 2 - 3 } & RA without ILD & RA with ILD & & \\
\hline FEV1/FVC\% & $91.13 \pm 9.51$ & $96.11 \pm 13.53$ & -1.530 & 0.133 \\
\hline FVC & $87.85 \pm 11.35$ & $64.22 \pm 13.35$ & 6.679 & $<0.001$ \\
\hline FEV1 & $80.02 \pm 13.58$ & $61.72 \pm 16.49$ & 4.261 & $<0.001$ \\
\hline
\end{tabular}

There was highly statistically significant difference between RA patients with and without ILD as regards to FVC and FEV1. 


\section{DISCUSSION}

Autoantibodies are highly specific markers for RA and are useful for predicting RA development and progression ${ }^{(\mathbf{1 0})}$.

Although the association between anti-CCP antibodies and extra-articular manifestations was not conclusive. Currently, there are few studies evaluating the association between anti-CCP autoantibodies and ILD in RA. Therefore, our aim in this study was to examine the relationship between serum levels of antiCCP3 and the various manifestations of rheumatoid arthritis-associated lung disease, as well as the effect of concurrent MTX treatment in those cases.

We studied 50 RA patients, HRCT chest of all patients showed 27(54\%) patients with normal HRCT, 23(46\%) with abnormal HRCT, As regards to clinical data for patients with abnormal HRCT. 15patients (65\%) has chest symptoms, 8 patients (34\%) did not show any chest symptoms. This was in agreement with Olson et al. ${ }^{(11)}$ and Doyle et al. ${ }^{(12)}$ who estimated that $30 \%$ of patients with rheumatoid arthritis have subclinical ILD noted on HRCT scans.

Habib et al. ${ }^{(13)}$ was agreed with us as they found that on a study conducted in 40 patients $27 \%$ of RA patients have abnormal HRCT finding despite absence of clinical symptoms.

Another Prospective study was done by Zrour et al. ${ }^{(14)}$ found that HRCT findings of 75 RA patients were abnormal in $48.6 \%$ of the patients with no respiratory symptoms.

Among patients with completely normal HRCT, 7 patients $(25 \%)$ has chest symptoms, 20 patients $(74 \%)$ did not show any chest symptoms. Patients with normal HRCT despite presence of chest complaint in the form of mild dyspnea on exertion showed increased incidence of other comorbidities including hypertension, diabetes mellitus and anemia

Bari et al. ${ }^{(15)}$ who studied 40 RA patients also reported dyspnea in $12 \%$ of RA patients du to anemia without concomitant chest problem

HRCT chest of all patients showed 19 (38\%) patients with evidence of ILD, 8 patients (16\%) with pulmonary nodules.

The reported prevalence of ILD in patients with RA is highly variable and depends on the methods of detection (e.g. high-resolution CT [HRCT] scan, chest radiograph, or pulmonary function testing) and the population selected for study (e.g. symptomatic or asymptomatic, autopsy series).

Carmona et al. ${ }^{(16)}$ who studied788 patients. Showed low incidence of interstitial lung disease 3.7\% (2.4 to 5.0), which is explained by the method of testing as they used chest X-ray only in addition to clinical examination.

Mori et al. ${ }^{(17)}$ performed high-resolution computed tomography (HRCT) on a total of 126 patients with early RA $(\mathrm{n}=65)$ and longstanding RA (n $=61$ ) showed $51 \%$ incidence of ILD,

Affaraa et al. ${ }^{\left({ }^{(18)}\right.}$ revealed that $38.3 \%(23 / 60)$ of all included RA patients had RA-ILD, while $61.7 \%$ (37/60) did not have ILD (RA-no ILD). All RA-ILD cases had high-resolution CT abnormalities characteristic of ILD.

Comparing RA patients in our study with and without ILD according to clinical data there was statistically significant difference between RA patients with andwithout ILD as regards to DAS 28, this was in agreement with Bongartz et al. ${ }^{(\mathbf{9 9})}$ who concluded that risk factors which had a statistically significant association with development of ILD were related to markers of disease activity

This was in addition to Rocha-Muñoz et al. (20) who studied 39 RA-ILD patients confirmed by highresolution computed tomography (HRCT) were compared with $42 \mathrm{RA}$ without lung involvement (RA only)and concluded also that DAS28, were associated with an increased risk for RA-ILD.

However, this was in contrary with Kelly $\boldsymbol{e t}$ al. (21) where they demonstrated that disease activity scores (DAS28) may be lower in RA patients with severe ILD than in those without at presentation at least initially. However, after 5 years of follow-up there were no differences in DAS28 scores between survivors with and without ILD.

Anti-CCP3 was positive in 30 patients $(60 \%)$ and negative in 20 patients $40 \%$, Anti-CCP titer ranged from 3 to 417 mean [173.62 \pm 180.82 ]. with sensitivity of about $60 \%$, which was in agreement with the systematic review done by Taylor et al. ${ }^{(5)}$, who assesses the current status of anti-cyclic citrullinated peptide (anti$\mathrm{CCP}$ ) test in 85 study contracting the diagnosis and prognosis of rheumatoid arthritis (RA).

In our study there was statistically significant difference between patients with and without ILD in HRCT as regards to anti-CCP titer, this was in agreement with Nikiphorou $\boldsymbol{e t}$ al. ${ }^{(22)}$ who recently demonstrated, their results of a multicenter study in which anti-CCP antibodies were strongly associated with ILD in RA.

Yin et al. ${ }^{(23)}$ identified ILD in 71 from among their 285 patients with RA, observing that positivity for second-generation anti-CCP (anti-CCP2) was associated with an increase in risk of ILD.

Kelly et al. (21) from a multicenter study, identified 230 patients with proven ILD in RA. These authors identified that anti-CCP antibody titers comprised the most relevant factor associated with ILD in RA.

Reynisdottir et al. ${ }^{(24)}$ employing a different approach, analyzed the findings of high-resolution computed tomography (HRCT) in 71 patients with early, untreated RA who were positive for 
anticitrullinated proteins (anti-CCP -positive)compared4with 35 patients with early, untreated anti-CCP negative RA. These authors identified that $63 \%$ of patients with anti-CCP -positive RA had abnormalities in HRCT compared with $37 \%$ of patients with ACPA-negative 5- If RA.

However Inui et al. ${ }^{(25)}$ on evaluating relatively small number of patients (18 patients), did not find an association between anti-CCP and ILD.

In our study also there was statistically significant difference between patients with and without ILD in HRCT as regards to FEV1, FVC, except for FEV1/FVC between patients with and without ILD in HRCT, forced expiratory volume in first second (FEV1), and forced vital capacity (FVC) are often decreased because of the reduction in lung volume, but the FEV1-to-FVC ratio is maintained or increased in ILD. Which agreed with Martinez ${ }^{(26)}$ and Affaraa et al. (18) who studied 60 RA patients and stated that FVC showed a trend toward a negative association with ILD.

\section{CONCLUSION}

1- The prevalence of ILD depends on clinical phenotype (e.g. the presence or absence of respiratory symptoms), duration of disease, clinical severity of RA, autoantibody profile, history of treatment, and the methods of diagnosis. Early studies utilizing chest radiographs found low prevalence of ILD, Subsequent studies screened patients irrespective of symptoms and have found higher prevalence.

2- RA patients without chest symptoms should not be neglected, pulmonary function testing and HRCT should be done routinely to pick up early ILD cases.

3- HRCT has fundamental role in differentiating ILD pattern in RA patients which have golden role in differentiating pathological patterns either due to pulmonary drug toxicity or disease induced ILD.

4- Higher anti-CCP3 titers are potentially associated with increased risk of developing interstitial lung disease in rheumatoid arthritis patients, and to be considered as markers of severity and extent of RA-ILD in HRCT.

5- MTX induced pulmonary toxicity is potentially reversible with discontinuation of MTX administration.

\section{RECOMMENDATIONS}

1- RA patients is risky for development of ILD, HRCT should be considered as routine in patients with RA either symptomatic or not symptomatic to early detect ILD or drug induced pulmonary toxicity.

2- Anti-CCP3 should be done routinely as a prognostic marker for RA-ILD.

3- Pulmonary function testing is considerd as routine for RA patients to monitor pulmonary functional performance.
Development of pathological HRCT changes in the form of ground glass opacity with or without reticulations in RA patients suggesting NSIP HRCT pattern either due to drug toxicity or disease pathology. ff patient is on MTX it should be stopped as its considered drug induced ILD.

\section{REFERENCES}

1. Young A, Koduri G (2007): Extra-articular manifestations and complications of rheumatoid arthritis. Best Pract Res Clin Rheumatol., 21(5):907-927.

2. Shaw M, Bridget FC, Lawrence AH et al. (2015): Rheumatoid arthritis-associated lung disease. European Respiratory Review, 24: 1-16.

3. Malik S, Saravanan V, Kelly C (2012): Interstitial lung disease in rheumatoid arthritis: an update on diagnosis and management. Intern J Clin Rheumatol., 7 (3): 297-308.

4. Cavagna L, Monti S, Grosso V et al. (2013): The multifaceted aspects of interstitial lung disease in rheumatoid arthritis. Biomed Res Int., 2013: 759-760.

5. Taylor P, Gartemann J, Hsieh J et al. (2011): A systematic review of serum biomarkers anti-cyclic citrullinated Peptide and rheumatoid factor as tests for rheumatoid arthritis. Autoimmune Diseases, 18: 815-38.

6. Aletaha D, Neogi T, Silman AJ et al. (2010): Rheumatoid arthritis classification criteria: an American College of Rheumatology/European League Against Rheumatism collaborative initiative. Arthritis Rheum., 62:2569-81.

7. van Riel PL, Barrera P, Laan RF et al. (1994): Methotrexate-related pulmonary complications in rheumatoid arthritis. Ann Rheum Dis., 53: 434-439.

8. Prevoo ML, van Gestel AM, van T et al. (1996): Remission in a prospective study of patients with rheumatoid arthritis. American Rheumatism Association preliminary remission criteria in relation to the disease activity score. Br J Rheumatol., 35:1101-5.

9. Cho O, Oh YT, Chun M et al. (2018): Prognostic implication of FEV1/FVC ratio for limited-stage small cell lung cancer. J Thorac Dis., 10(3):1797-1805.

10.Korkmaz C, Us T, Kaşifoğlu T et al. (2006): Anti-cyclic citrullinated peptide (CCP) antibodies in patients with long-standing rheumatoid arthritis and their relationship with extra-articular manifestations. Clinical Biochemistry, 39(10):961-965.

11. Olson AL, Swigris JJ, Sprunger DB et al. (2011): Rheumatoid arthritis-interstitial lung disease-associated mortality. Am J Respir Crit Care Med., 183: 372-378.

12. Doyle TJ, Lee JS, Dellaripa PF et al. (2014): A roadmap to promote clinical and translational research in rheumatoid arthritis-associated interstitial lung disease. Chest, 145: 454-463.

13. Habib HM, Eisa AA, Arafat WR et al. (2011): Pulmonary involvement in early rheumatoid arthritis patients. Clin Rheumatol., 30: 217-221.

14.Zrour SH, Touzi M, Bejia I et al. (2005): Correlations between high-resolution computed tomography of the chest and clinical function in patients with rheumatoid arthritis. Joint Bone Spine, 72(1):41-7.

15. Bari MA, Sutradhar SR, Sarker CN et al. (2013): Assessment of anaemia in patients with rheumatoid arthritis. Mymensingh Med J., 22(2):248-54. 
16. Carmona L, González-Alvaro I, Balsa A et al. (2003): Rheumatoid arthritis in Spain: occurrence of extra-articular manifestations and estimates of disease severity. Ann Rheum Dis., 62(9):897-900.

17. Mori S, Cho I, Koga Y et al. (2008): Comparison of pulmonary abnormalities on high-resolution computed tomography in patients with early versus longstanding rheumatoid arthritis. J Rheumatol., 35(8):1513-21.

18. Affaraa NK, Refaata AM, Elgawishb MH et al. (2016): High-resolution CT and pulmonary function tests in rheumatoid arthritis patients with subclinical interstitial lung disease in Kuwait. The Egyptian Rheumatologist, 38(2):77-83.

19. Bongartz T, Nannini C, Medina-Velasquez YF et al. (2010): Incidence and mortality of interstitial lung disease in rheumatoid arthritis: a population-based study. Arthritis Rheum., 62(6):1583-1591

20. Rocha-Muñoz AD, Ponce-Guarneros M, Gamez-Nava JI et al. (2015): Anti-cyclic citrullinated peptide antibodies and severity of interstitial lung disease in women with rheumatoid arthritis. J Immunol Res., 2015: 151-62.

21. Kelly CA, Saravanan V, Nisar M et al. (2014): Rheumatoid arthritis-related interstitial lung disease: associations, prognostic factors and physiological and radiological characteristics - a large multicentre UK study. Rheumatology (Oxford), 53(9):1676-1682.

22. Nikiphorou E, Chan E, Saravanan V et al. (2013): Serological biomarkers for the development of rheumatoid arthritis related interstitial lung disease. Arthritis and Rheumatology, 65(10): 423-26.

23. Yin Y, Liang D, Zhao L et al. (2014): Anti-cyclic citrullinated peptide antibody is associated with interstitial lung disease in patients with rheumatoid arthritis. doi: 10.1371/journal.pone.0092449.e92449

24. Reynisdottir G, Karimi R, Joshua V et al. (2014): Structural changes and antibody enrichment in the lungs are early features of anti-citrullinated protein antibody-positive rheumatoid arthritis. Arthritis and Rheumatology, 66(1):31-39.

25.Inui N, Enomoto N, Suda T et al. (2008): Anti-cyclic citrullinated peptide antibodies in lung diseases associated with rheumatoid arthritis. Clinical Biochemistry, 41(13):1074-1077.

26. Martinez FJ (2006): Idiopathic interstitial pneumonias: usual interstitial pneumonia versus nonspecific interstitial pneumonia. Proc Am Thorac Soc., 3: 81-95. 